\title{
Conhecimento populacional sobre leishmaniose no município de Marília, São Paulo, Brasil
}

\author{
Population awareness of Leishmaniasis in the municipality of Marilia, \\ São Paulo, Brazil
}

Amanda Garcia Youssef (D), Luiz Liutti Netto (D)*, Bruno Roberto Vidal Tuani (D), Janayna Maria Parente Serafim (D), Daniel De Bortoli Teixeira (D), Elma Pereira dos Santos Polegato, Camila Dias Porto (D), Milena Friolani (D)

Universidade de Marília (UNIMAR), Marília, SP, Brasil

\section{Resumo}

A leishmaniose constitui um grupo de doenças infecciosas não contagiosas causadas por protozoários do gênero Leishmania. Possui, principalmente, apresentações viscerais e cutâneas. Diferentes formas da doença são amplamente distribuídas em todo o mundo, constituindo um importante problema de saúde pública. 0 cão doméstico (Canis familiaris) é apontado epidemiologicamente como o reservatório mais importante para Leishmania infantum, cuja transmissão ocorre por meio de flebotomíneos infectados com o protozoário. A falta de informação e de atitudes preventivas são alguns dos principais fatores relacionados à persistência das doenças infecciosas e parasitárias no Brasil. No caso das leishmanioses, na maioria das áreas onde a doença é endêmica o conhecimento restringese, muitas vezes, às pessoas que já tiveram a doença ou a casos na família ou proximidades. Por meio de inquéritos é possível buscar e detectar necessidades invisíveis, atuando com instrumento que permita, em primeiro plano, identificar e priorizar necessidades sanitárias por meio de amostras representativas de determinada população. Neste estudo, objetivou-se avaliar o conhecimento da população do município de Marília sobre leishmaniose, buscando identificar as particularidades da enfermidade que necessitam de maiores esclarecimentos. 0 estudo foi realizado por meio de inquérito populacional, com questões fechadas sobre o tema leishmaniose, abordando conceitos a respeito do conhecimento da enfermidade, formas de transmissão, agente etiológico, vetor e sintomatologia da doença. Do total de 420 pessoas entrevistadas, 91,19\% afirmaram conhecer a doença, porém apenas 69,71\% destas conheciam de fato o vetor/forma de transmissão da enfermidade e $22,45 \%$ tinham conhecimento sobre onde o vetor se multiplica. Dos entrevistados que afirmaram conhecer a doença, 83,29\% apontaram que humanos também podem adquirir a afecção e 40,99\% disseram que cães podem ser curados. Os resultados demonstram que 
apesar de a maior parte da população de Marília afirmar conhecer a leishmaniose, ainda existe insuficiência de informações em alguns aspectos, frisando a necessidade de melhorias na divulgação de particularidades da enfermidade.

Palavras-chave: Epidemiologia. Protozoose. Zoonose. Saúde pública.

\section{Abstract}

Leishmaniasis is a group of non-contagious infectious diseases caused by protozoa of the Leishmania genus. The disease has two main forms, which are visceral and cutaneous. Different forms of Leishmaniasis are widely spread throughout the world, making this disease a major public health problem. Domesticated dogs (Canis familiaris) are epidemiologically identified as the most important Leishmania infantum reservoir, which is transmitted by the bite of infected phlebotomine sandflies. The lack of information and preventive measures are some of the main factors related to the persistence of infectious and parasitic diseases in Brazil. In most areas where Leishmaniasis is endemic, the awareness regarding this disease is restricted to residents who either have had the disease or heard about cases in their families or in the neighborhood. The use of surveys enables the search and detection of invisible needs, by using an instrument that primarily identifies and prioritizes health needs, through representative samples of a certain population. This study aimed to evaluate the population awareness of leishmaniasis in the municipality of Marilia, seeking to identify the particularities of the disease that need further enlightenment. The study was carried out through a population survey, with closed questions about leishmaniasis, addressing concepts regarding the disease awareness, transmission modes, etiologic agent, vector and symptomatology. Out of the 420 people interviewed during the survey, $91.19 \%$ were aware of the disease but $69.71 \%$ actually knew about the disease vector and transmission modes, and 22,45\% knew where the vector multiplied. Out of the respondents who were aware of the disease, 83.29\% answered that humans can also acquire the condition and 40.99\% answered that dogs may be cured. The results show that although most of the population in Marília claims to be aware of leishmaniasis, people still have insufficient knowledge about some aspects of the disease, which reveals the need for improvements in the awareness campaigns of the disease.

Keywords: Epidemiology. Protozoosis. Public health. Zoonosis.

\section{Introdução}

A leishmaniose constitui um grupo de doenças infecciosas não contagiosas, causadas por protozoários do gênero Leishmania, e possui principalmente apresentações viscerais e cutâneas. Diferentes formas da doença são amplamente distribuídas em todo o mundo e são endêmicas em 88 países, em quatro continentes, constituindo um importante problema de saúde pública devido à sua incidência e alta letalidade (Alvar et al., 2012; Benitez et al., 2018).

A leishmaniose é considerada uma das mais importantes doenças zoonóticas transmitidas por vetores, com alta taxa de mortalidade em humanos se não tratada (Asfaram et al., 2017).

A leishmaniose visceral é a forma mais grave de leishmaniose, sendo o cão doméstico (Canis familiaris) considerado epidemiologicamente o reservatório mais importante para Leishmania infantum, cuja transmissão ocorre por meio de flebotomíneos infectados com o protozoário (Nunes et al., 2015). Comumente, cães acometidos apresentam linfadenomegalia, esplenomegalia, atrofia muscular, perda de peso, alopecia, onicogrifose, hiporexia, letargia, além de lesões cutâneas (Noli e Saridomichelakis, 2014). Todos esses sinais clínicos aparecem em proporções variáveis em cães sintomáticos, porém grande parte dos animais afetados não apresenta sintomatologia clínica, dificultando o diagnóstico e facilitando a transmissão da enfermidade (Reguera et al., 2016).

As leishmanioses são consideradas doenças negligenciadas pelo fato de não disporem de tratamentos eficazes ou adequados, e por afetarem milhões de pessoas ao redor do mundo, sobretudo indivíduos carentes e residentes em países em desenvolvimento, como o Brasil (Brito et al., 2015).

Mesmo com grande parte dos casos subnotificados, atualmente o Brasil é responsável por 
cerca de 90\% dos casos humanos da América Latina (Castro et al., 2016). A falta de informação e de atitudes preventivas são alguns dos principais fatores relacionados à persistência das doenças infecciosas e parasitárias no país. No caso das leishmanioses, na maioria das áreas onde a doença é endêmica o conhecimento restringe-se, muitas vezes, às pessoas que já tiveram a doença ou a casos na família ou vizinhos (Uchôa et al., 2004).

A eficiência da participação da população como agentes multiplicadores da informação em ações de prevenção e controle de doenças, inclusive da leishmaniose, já foi comprovada (Lobo et al., 2013). Por meio de inquéritos é possível buscar e detectar necessidades invisíveis, atuando como instrumento que permita, em primeiro plano, identificar e priorizar necessidades sanitárias por meio de amostras representativas de determinada população (Tome et al., 2010)

0 presente estudo objetiva avaliar o conhecimento da população do município de Marília, SP, a respeito da leishmaniose, buscando identificar particularidades da enfermidade que necessitem de maior elucidação.

\section{Material e métodos}

A pesquisa foi realizada por meio de inquérito populacional, durante campanha de vacinação antirrábica, com tutores se apresentando nos postos de vacinação. 0 estudo respeitou os princípios éticos estabelecidos pela Resolução ${ }^{\circ}$ 466/12 do Conselho Nacional de Saúde, e foi aprovado pelo Comitê de Ética em Pesquisa da Universidade de Marília, sob o parecer $\mathrm{n}^{\circ}$ 2.996.738.

As questões, de múltipla escolha, abordaram conceitos a respeito do conhecimento da enfermidade, formas de transmissão, agente etiológico, sintomatologia da doença, além de informações socioeconômicas como renda salarial e grau de instrução escolar.

Para o cálculo da amostra necessária para representar a população do município de Marília, foram considerados os seguintes parâmetros estatísticos: população estimada de 237.130 habitantes (IBGE, 2018), nível de confiança de $95 \%$ e erro amostral de 5 pontos percentuais. Com base nessas informações, a amostra mínima para esse estudo foi estimada em 384 participantes, no entanto foram entrevistadas 420 pessoas, número acima do requerido.

Para avaliar a associação das variáveis categóricas foi utilizado o teste de Qui-quadrado, com 95\% de confiança. Todas as análises foram conduzidas no software R (R Core Team, 2017).

\section{Resultados e discussão}

Dos 420 indivíduos entrevistados, 55,95\% eram mulheres e $44,05 \%$ homens. Verificou-se que a maior parte dos entrevistados (24,29\%) tinha entre 18 e 25 anos, e $23,33 \%$ tinham idade acima de 55 anos. A maioria dos entrevistados possuía ensino médio completo $(34,52 \%)$ e apenas $1,19 \%$ não possuía escolaridade (Tabela 1).

Tabela 1 - Grau de escolaridade dos tutores entrevistados de acordo com sexo

\begin{tabular}{ccccccc}
\hline & \multicolumn{2}{c}{ Feminino } & \multicolumn{2}{c}{ Masculino } & \multicolumn{2}{c}{ Total } \\
\cline { 2 - 7 } & $\mathrm{n}$ & $\%$ & $\mathrm{n}$ & $\%$ & $\mathrm{n}$ & $\%$ \\
\hline Sem escolaridade & 4 & 0,95 & 1 & 0,24 & 5 & 1,19 \\
$\begin{array}{c}\text { Ensino fundamental } \\
\text { incompleto }\end{array}$ & 27 & 6,43 & 27 & 6,43 & 54 & 12,86 \\
$\begin{array}{c}\text { Ensino fundamental } \\
\text { completo }\end{array}$ & 15 & 3,57 & 18 & 4,29 & 33 & 7,86 \\
$\begin{array}{c}\text { Ensino médio } \\
\text { incompleto }\end{array}$ & 16 & 3,81 & 12 & 2,86 & 28 & 6,67 \\
Ensino médio & 73 & 17,38 & 72 & 17,14 & 145 & 34,52 \\
completo & & & & & & \\
Ensino superior & 48 & 11,43 & 19 & 4,52 & 67 & 15,95 \\
incompleto & & & & & & \\
Ensino superior & 46 & 10,95 & 30 & 7,14 & 76 & 18,10 \\
$\quad$ completo & & & & & & \\
Pós-graduação & 6 & 1,43 & 6 & 1,43 & 12 & 2,86 \\
\hline Total geral & 235 & 55,95 & 185 & 44,05 & 420 & 100,00 \\
\hline
\end{tabular}

Verificou-se que $91,19 \%$ dos 420 tutores entrevistados relataram conhecer a enfermidade, sendo este um dado positivo. Anversa et al. (2016), em pesquisa na cidade de Bauru, SP, relataram que 99,3\% das pessoas já ouviram falar da doença, índice que pode ser influenciado pelo fato de Bauru 
ser uma das cidades mais afetadas do estado de São Paulo (Ortiz e Anversa, 2015). Paulan et al. (2016), em inquérito com famílias de um assentamento rural no município de Ilha Solteira, SP, descreveram que $77 \%$ das mesmas sabiam o que é leishmaniose. Já Camargo e Bondan (2015), em estudo realizado junto à população de Cotia, SP, observaram que 98,6\% dos indivíduos entrevistados não possuíam qualquer conhecimento sobre Leishmaniose, indicando um nível de conhecimento da população local muito baixo acerca da doença e diferindo completamente dos resultados observados no presente estudo.

Quanto à distribuição de pessoas que sabiam qual a forma de transmissão da doença, no presente trabalho $64,29 \%$ relataram ser o mosquito-palha, porém 9,52\% relataram que o cachorro era o responsável pela transmissão, dado compatível com os encontrados por Anversaet al. (2016), em que $77,1 \%$ dos entrevistados responderam que a transmissão da enfermidade ocorre pela picada de flebotomíneos e 13,6\% pelo cão infectado. Guimarães (2013) observou que $92 \%$ dos entrevistados conheciam a forma de transmissão da doença, enquanto Lima et al. (2008) observaram que 93,7\% dos entrevistados apontaram o mosquito como vetor. Já Porto et al. (2015) descreveram que $40,55 \%$ dos entrevistados afirmaram ser o cão o responsável pela transmissão da enfermidade.

Questionados sobre o local de reprodução do vetor, $28,48 \%$ dos participantes relataram ser em folhas secas, $26,67 \%$ em água parada, 3,57\% em bueiros, 1,9\% em cavernas e 47,38\% não souberam responder. Folhas secas representam substrato propício para a reprodução do Lutzomya longipalpis, vetor da leishmaniose (Meirelles et al., 2018). Paulan et al. (2016), em pesquisa realizada em área rural do município de Ilha Solteira, SP, relataram que $58,97 \%$ de seus entrevistados sabiam qual o habitat do vetor, índice consideravelmente superior ao da presente pesquisa. Este contraste de resultados pode ser explicado pelo fato de que $1 / 4$ da população canina estudada pelos autores apresentava sorologia positiva para a enfermidade.

Em relação ao período do dia de aparecimento do vetor, $10,95 \%$ relataram ser durante a manhã,
$13,33 \%$ pelo entardecer, $7,62 \%$ durante à noite e $68,1 \%$ não souberam responder. 0 vetor da leishmaniose normalmente apresenta maior atividade ao entardecer, realizando o repasto sanguíneo entre a segunda e a quinta hora após o pôr do sol (Mayo et al., 1998).

Observou-se que os principais sinais clínicos de leishmaniose reportados pelos tutores foram emagrecimento $(35,05 \%)$, queda de pelo $(30,71 \%)$ e feridas pelo corpo (22,38\%), resultados corroborados por estudos realizados por Moura et al. (2015) em população de três bairros de Teresina, PI, em que constaram os sinais clínicos de leishmaniose mais relatados: emagrecimento (38\%), queda de pelos $(57,3 \%)$ e feridas na pele (40,6\%). Brustoloni et al. (2013), ao realizarem questionário com familiares de crianças portadoras de leishmaniose visceral, observaram que $64,2 \%$ dos familiares souberam referir um ou mais sinais clínicos no cão, incluindo emagrecimento, lesões cutâneas e crescimento anormal das unhas. Porto et al. (2015), por sua vez, mencionam que os sinais clínicos mais relatados são diarreia, febre e emagrecimento, sendo apontados por $56,66 \%$ dos entrevistados.

Verificou-se que os tutores não possuem um conhecimento adequado quanto ao tratamento da enfermidade nos cães, visto que 40,24\% afirmaram que a leishmaniose tem cura, 20,0\% disseram não saber e 39,76\% afirmaram não existir cura. 0 Ministério da Agricultura, Pecuária e Abastecimento e o Ministério da Saúde aprovaram conjuntamente o uso da miltefosina (Milteforam ${ }^{\circledR}$ ) para o tratamento de leishmaniose visceral canina (MAPA, 2016), porém, apesar dos resultados favoráveis, a miltefosina não promove a cura da enfermidade, podendo o hospedeiro ainda portar o agente etiológico da doença, servindo como um reservatório para a manutenção da enfermidade (Andrade et al., 2011; Henriques, 2016). Dados obtidos por Massia (2017) divergem da presente pesquisa, onde $10 \%$ dos entrevistados disseram haver cura, $78 \%$ relataram não haver cura e 12\% desconheciam a informação. Essa diferença de resultados se deve ao fato de a pesquisa realizada por Massia (2017) envolver profissionais da saúde como médicos, veterinários, enfermeiros e agentes comunitários. 
Em relação à possibilidade de transmissão dos cães para seres humanos, $80 \%$ dos tutores relataram que humanos podem adquirir leishmaniose, $7,62 \%$ afirmaram que a enfermidade não pode ser transmitida e $12,38 \%$ não souberam responder. Resultados condizentes com a presente pesquisa foram descritos por Guimarães (2013), em que 89,55\% dos entrevistados responderam que a doença pode ser contraída por seres humanos. Já Ribeiro (2010), em pesquisa com professoras do ensino fundamental, buscando o conhecimento sobre zoonoses diversas, obteve resultado preocupante, uma vez que somente $22,5 \%$ das entrevistadas reconheciam a leishmaniose como uma zoonose. Oliveira-Neto et al. (2018), em pesquisa realizada em um hospital veterinário com tutores de cães, relatam que $23 \%$ dos entrevistados afirmaram que a leishmaniose se trata de uma zoonose. Costa et al. (2017), em pesquisa avaliando o conhecimento sobre zoonoses de agentes comunitários e professores, verificaram que $25,7 \%$ dos entrevistados tinham um nível de conhecimento considerado bom pelos autores, $60 \%$ tinham conhecimento regular e $14,3 \%$, conhecimento insuficiente.

Quanto a terem recebido orientação de agente de saúde pública sobre a leishmaniose, abordando conceitos de transmissão, vetor, reservatório e formas de profilaxia, $37,86 \%$ disseram que foram orientados sobre tais informações e $62,14 \%$ relataram nunca terem sido informados. Questionados sobre qual seria a melhor forma de a informação chegar até a população, a maior parte dos tutores entrevistados mencionou a internet $(38,09 \%)$, seguida da televisão $(27,28 \%)$ e de agentes de saúde pública $(24,28 \%)$. Langoni et al. (2014), em pesquisa no município de Botucatu, SP, relataram que $84,8 \%$ da população utiliza programas televisivos como forma de obtenção de informação. Atualmente os meios de comunicação passaram a ter um caráter informativo e educacional, com a viabilidade de comunicação rápida e abrangente que antes provinha apenas por meio das famílias e escolas. Neste contexto, as mídias têm um papel importante de veiculação de informações relativas à saúde populacional, tornando-se uma forma de conscientização (Cavaca, 2015).
Embora apenas 38,6\% dos tutores entrevistados afirmarem já terem recebido orientações de agentes de saúde, 91,2\% afirmam conhecer a enfermidade. Destes, $69,7 \%$ possuem conhecimento sobre o vetor e $22,5 \%$ sobre o local de reprodução do mesmo. Além disso, 83,3\% possuem conhecimento sobre a transmissibilidade da enfermidade a humanos e $41,0 \%$ sobre a possibilidade de cura.

Já em relação à afirmação do conhecimento sobre leishmaniose e fatores socioeconômicos, inexiste a associação com escolaridade $(p=0,41)$, idade $(p=0,24)$, sexo $(p=0,75)$, renda $(p=0,15)$ e região de habitação na cidade (zonas norte, sul, leste, oeste e centro) ( $p=0,99)$. Possivelmente, essa não correlação se deve a uma divulgação que atinge todas as camadas sociais da população de Marília. Guimarães (2013), em seu estudo, também relata que não houve relação significativa entre os níveis de escolaridade e sexo dos entrevistados $(\mathrm{p}>0,05)$.

\section{Conclusão}

De forma geral, nota-se que a população entrevistada no presente estudo possui um maior conhecimento em relação a aspectos básicos da doença, como reconhecimento do vetor e classificação da leishmaniose como zoonose. Já em relação a conhecimentos mais específicos e que impactam diretamente a profilaxia da enfermidade, como local de proliferação e hábitos do vetor (período de maior atividade, por exemplo), além dos sinais clínicos em cães, o público entrevistado demonstrou estar pouco informado.

Aspectos como o fato de grande parte dos entrevistados não assimilar que o animal que recebe o tratamento para a enfermidade ainda possa ser portador da protozoonose e contribuir para a permanência da doença na região são preocupantes e necessitam ser melhor elucidados para a população.

Por fim, ressalta-se a necessidade da excelência em educação em saúde, visto que o conhecimento consolidado pela população exerce grande parte de medidas profiláticas para o controle da enfermidade. 


\section{Referências}

Alvar J, Vélez ID, Bern C, Herrero M, Desjeux P, Cano J, et al. Leishmaniasis worldwide and global estimates of its incidence. PloS One. 2012;7(5):e35671.

Andrade HM, Toledo VPCP, Pinheiro MB, Guimarães TMPD, Oliveira NC, Castro JA, et al. Evaluation of miltefosine for the treatment of dogs naturally infected with L. infantum (= L. chagasi) in Brazil. Vet Parasitol. 2011;181(2-4):83-90.

Anversa L, Montanholi RJD, Sabino DL. Avaliação do conhecimento da população sobre leishmaniose visceral. Rev Inst Adolfo Lutz. 2016;75:1685.

Asfaram S, Fakhar M, Soosaraei M, Teshnizi SH, Mardani A, Banimostafavi ES, et al. Global status of visceral leishmanial infection among blood donors: A systematic review and meta-analysis. Transfus Apher Sci. 2017;56(5):748-54.

Benitez NA, Martins FDC, Mareze M, Nino BSL, Caldart ET, Ferreira FP, et al. Spatial and simultaneous seroepidemiology of anti-Leishmania spp. antibodies in dog owners and their dogs from randomly selected households in a major city of southern Brazil. Prev Vet Med. 2018;154:47-53.

Brito JA, Santos RA, Mendonça BC, Ribeiro RR. Avaliação do conhecimento sobre a leishmaniose visceral antes e depois de intervenção educacional em proprietários de cães da cidade de Cruz das Almas, Recôncavo da Bahia. Rev Cienc. Ext. 2015;11(2):104-14.

Brustoloni FM, Serra JPA, Souza AB, Pereira EML, Machado LBP, Branco NM, et al. Aspectos socioeconômicos e conhecimento de familiares de crianças acometidas pela Leishmaniose visceral no Mato Grosso do Sul. Ensaios Cienc Biol Agrar Saude. 2013;17(3):71-82.

Camargo TC, Bondan EF. Conhecimentosobreleishmaniose visceral canina na população do município de Cotia (SP), Brasil, e participação dos clínicos veterinários locais na propagação de medidas preventivas. R Bras Ci Vet. 2015;22(1):28-33.
Castro JM, Rodrigues SM, Tarso S, Costa FL, Rodrigues ACCP, Vieira LDF, et al. Conhecimento, percepções de indivíduos em relação à leishmaniose visceral humana como novas ferramentas de controle. Ensaios Cienc Biol Agrar Saude. 2016;20(2):93-103.

Cavaca AG. Doenças midiaticamente negligenciadas: cobertura e invisibilidade de temas sobre saúde na mídia impressa [tese]. Rio de Janeiro: Escola Nacional de Saúde Pública Sergio Arouca; 2015.177p.

Costa GJA, Cavalcanti SMA, Lins Filho NM, Della Bella S, Marvulo MFV, Bezerra R, et al. Avaliação da percepção sobre zoonoses com agentes de saúde, combate a endemias e docentes de escola públicas, do entorno da Estação Ecológica de Caetés, Região Metropolitana do Recife - PE, Brasil. Med Vet (UFRPE). 2017;11(1):67-75.

Guimarães ELAM. Conhecimento sobre leishmaniose visceral e prática das medidas de prevenção e controle por proprietários de cães em Belo Horizonte [dissertação]. Belo Horizonte: Universidade Federal de Minas Gerais; 2013. 82 p.

Henriques MIC. Avanços recentes na terapêutica da Leishmaniose Canina e na toxicidade dos fármacos usados [tese]. Covilhã: Universidade da Beira Interior; 2016.120 p.

IBGE. População estimada. Diretoria de Pesquisas, Coordenação de População e Indicadores Sociais. Estimativas da população residente com data de referência $1^{\text {o }}$ de julho de 2018. [acesso 31 out 2018]. Disponível em: https://tinyurl.com/yy9j6j3g.

Langoni H, Troncareli MZ, Rodrigues EC, Nunes HRC, Lucheis SB, Victória $\mathrm{C}$, et al. Inquérito sobre o conhecimento de zoonoses relacionadas a cães e gatos em Botucatu-SP. Vet e Zootec. 2014 21(2):297-305.

Lima FF, Koivisto MB, Perri SHV, Bresciani KDS. O conhecimento de idosos sobre parasitoses em instituições não governamentais do município de Araçatuba, SP. Rev Cienc Ext. 2008;4(1):77-85.

Lobo KS, Bezerra JMT, Brito LMO, Silva JS, Pinheiro VCS. Conhecimentos de estudantes sobre Leishmaniose Visceral em escolas públicas de Caxias, Maranhão, Brasil. Cienc Saude Coletiva. 2013;18(8):2295-300. 
Mapa. Nota Técnica Conjunta $\mathrm{n}^{\circ}$ 001/2016 MAPA/MS. 2016 [acesso 8 abr 2019]. Disponível em: https://tinyurl. com/y6dr6p4o.

Massia LI. Leishmaniose visceral: avaliação do conhecimento dos agentes de saúde pública em Uruguaiana (RS) [dissertação]. Uruguaiana: Universidade Federal do Pampa; 2017. 85 p.

Mayo RC, Casanova C, Mascarini LM, Pignatti MG, Rangel O, Galati EAB, et al. Flebotomíneos (Diptera, Psychodidae) de área de transmissão de leishmaniose tegumentar americana, no município de Itupeva, região sudeste do Estado de São Paulo, Brasil. Rev Soc Bras Med Trop. 1998;31(4):339-45.

Meirelles MH, Martins KA, Mota TF, Warburg A, Fraga DBM. Preferência de criadouros do flebotomíneo Lutzomya longipalpis e seu possível papel na transmissão de Leishmania. Anais MedTrop - 54 ${ }^{\circ}$ Congresso da Sociedade Brasileira de Medicina Tropical; 2-5 set 2018; Olinda, PE. Brasília: SBMT; 2018.

Moura LMA, Monte NDP, Sousa RLT, Santos JP, Freire SM. Nível dos conhecimentos sobre as leishmanioses visceral e tegumentar pela população de alguns bairros de Teresina, Piauí, Brasil. Cient Cienc Biol Saude. 2015;17(1):21-30.

Noli C, Saridomichelakis MN. An update on the diagnosis and treatment of canine leishmaniosis caused by Leishmania infantum (syn. L. chagasi). Vet J. 2014;202(3):425-35.

Nunes CM, Lima VMF, Melo GD, Paula HB, Perreira MEG, Tronco CMT, et al. Serological, parasitological and molecular tests for canine visceral leishmaniosis diagnosis in a longitudinal study. Rev Bras Parasitol Vet. 2015;24(4):402-9.

Oliveira-Neto RR, Souza VF, Carvalho PFG, Frias DFR. Nivel de conhecimento de tutores de cães e gatos sobre zoonoses. Rev Salud Publica. 2018;20(2):198-203.
Ortiz RC, Anversa L. Epidemiologia da leishmaniose visceral em Bauru, São Paulo, no período de 2004 a 2012: um estudo descritivo. Epidemiol Serv Saude. 2015;24(1):97-104.

Paulan SC, Silva DT, Lins AGS, Lima FL, Tenório MS, Tasca $\mathrm{KI}$, et al. 0 conhecimento sobre leishmaniose visceral: suficiente para controle e prevenção? Rev Cienc Ext. 2016;12(2);47-60.

Porto LS, Moura AFA, Moreira GAL, Naser HSHN, Mendonça VDF, Faria TA, et al. O conhecimento sobre as diferenças entre leishmaniose e dengue dos idosos acima de 60 anos do bairro Paracatuzinho, Paracatu - MG. Rev Cient Med Faculdade Atenas. 2015;9(1): 17-30.

R Core Team. R: A language and environment for statistical computing. Viena, Áustria: R Foundation for Statistical Computing; 2017.

Reguera RM, Morán M, Pérez-Pertejo Y, García-Estrada C, Balaña-Fouce R. Current status on prevention and treatment of canine leishmaniasis. Vet Parasitol. 2016;227:98-114.

Ribeiro LML. Análise do conhecimento, sobre leishmaniose visceral e outras zoonoses, de docentes dos três primeiros anos do ensino fundamental em escolas da Região Noroeste de Belo Horizonte, Minas Gerais [dissertação]. Belo Horizonte: Universidade Federal de Minas Gerais; 2010.113 p.

Tome RO, Langoni H, Peruca LCB, Babboni SD. Avaliação do conhecimento sobre algumas zoonoses com proprietários de cães da área urbana do município de Botucatu - SP. UNOPAR Cient Cienc Biol Saude. 2010;12(3):67-74.

Uchôa CMA, Serra CMB, Magalhães CM, Silva RMM, Figliuolo LP, Leal CA, et al. Educação em saúde: ensinando sobre a leishmaniose tegumentar americana. Cad Saude Publica. 2004;20(4):935-41. 